\section{Response to: 'Additional proposals to reduce comorbidity in patients with chronic inflammatory rheumatic diseases'. Screening for comorbidities: what is the remit of rheumatologists?}

We thank Castañeda et $a l^{1}$ for their interest in our paper ${ }^{2}$ and are pleased to have the opportunity to respond to their comments in order to clarify what part of the management of comorbidities we believe to be within the remit of rheumatologists, that is, what is likely to be done by the rheumatologist and what rheumatologists are able to do in the daily practice.

The European League Against Rheumatism (EULAR) task force anticipated that the screening of a wide scope of comorbidities would make the final process too complex or too extensive to be implemented. The EULAR task force acknowledged that fibromyalgia impacts on the assessment of the disease activity and response to treatment of chronic inflammatory rheumatic diseases (CIRDs). American College of Rheumatology 1990 classification criteria for fibromyalgia ${ }^{3}$ and recent recommendations ${ }^{4}$ considered fibromyalgia as a diagnosis of exclusion that requires a specific visit in order to rule out other disorders that could be the cause of symptoms and to evaluate fibromyalgia severity. Therefore, we considered that the screening for fibromyalgia was beyond the scope of this initiative.

Moreover, we strived to focus on the screening or the reporting of comorbidities for which specific and pragmatic recommendations could be made. Consequently, we mainly considered the screening of risk factors that are highly predictive of significant comorbidities. We agree that surrogate marker of obesity such as waist circumference are superior to body mass index in predicting cardiovascular risk. However, substantial evidence of ethnic, sex and age variations in waist circumference, ${ }^{5}$ hampering the collection of waist circumference by a rheumatologist in his/her daily practice. 25-hydroxyvitamin $\mathrm{D}$ has a dual benefit for prevention of fractures in the elderly: ${ }^{6}$ a benefit on bone density and muscle strength. However, the correlation between 25-hydroxyvitamin D levels and risk of fractures in younger patients, such as patients with spondyloarthritis, is still unclear. ${ }^{7}{ }^{8}$ Therefore, the EULAR task force did not recommend systematically assessing serum 25-hydroxyvitamin D in every patient with CIRDs.

Finally, we anticipated that next steps would be required to facilitate the dissemination of this initiative in European countries in accordance with national guidelines. These EULAR points to consider were recently disseminated in France, using an evidencebased approach followed by expert consensus. ${ }^{9}$ In this initiative, Gossec et al clearly defined what part of the management of comorbidities is potentially within the remit of French rheumatologists. Screening for asymptomatic atherosclerotic plaques by use of carotid ultrasound is likely to reclassify patients with rheumatoid arthritis as very high risk patients, as evidence of carotid plaques was reported in $65 \%$ patients at moderate total cardiovascular risk (HEART score risk 1-5\%). ${ }^{10}$ Thus, it was proposed to refer all patients with a risk $>1 \%$ because the decision to perform carotid ultrasound and also the interpretation of this examination and subsequent treatment decisions should be made by a specialist. In this initiative, ${ }^{9}$ a visit to the dentist was recommended every year, in view of both the infectious risk represented by poor dental hygiene, and potential links between some CIRDs and periodontitis, as suggested by Castañeda et $_{\text {al }}{ }^{1}$

In summary, the EULAR Task Force does recognise the interest of the evaluation of comorbidities in patients suffering from chronic inflammatory rheumatic diseases and is proposing a core set of domains and items to be evaluated. However, the implementation of these proposals has to consider not only the national and local recommendations in this area but also the medical problems for each individual patient.

\section{Athan Baillet, ${ }^{1}$ Laure Gossec, ${ }^{2}$ Maxime Dougados ${ }^{3}$}

${ }^{1}$ Department of Rheumatology, GREPI-UGA EA7408, Grenoble, France ${ }^{2}$ Department of Rheumatology, Sorbonne Universités, UPMC Univ Paris 06, Institut Pierre Louis d'Epidémiologie et de Santé Publique, GRC-UPMC 08 (EEMOIS); AP-HP, Pitié Salpêtrière Hospital, Paris, France

${ }^{3}$ Department of Rheumatology, Paris Descartes University, 一Hôpital Cochin. Assistance Publique-Hôpitaux de Paris. INSERM (U1153): Clinical Epidemiology and Biostatistics, PRES Sorbonne Paris-Cité, Paris, France

Correspondence to Dr Athan Baillet, Service de Rhumatologie CHU Grenoble, Av de Kimberley, Echirolles 38434, France; abaillet@chu-grenoble.fr

Competing interests None declared.

Provenance and peer review Commissioned; internally peer reviewed.

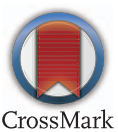

To cite Baillet A, Gossec L, Dougados M. Ann Rheum Dis 2016;75:e56.

Received 18 May 2016

Accepted 27 May 2016

Published Online First 14 June 2016

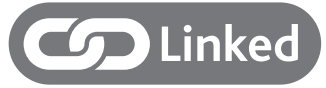

- http://dx.doi.org/10.1136/annrheumdis-2016-209836

Ann Rheum Dis 2016;75:e56. doi:10.1136/annrheumdis-2016-209861

\section{REFERENCES}

1 Castañeda S, Vicente EF, González-Gay MA. Additional proposals to reduce comorbidity in patients with chronic inflammatory rheumatic diseases: comment on 'Points to consider for reporting, screening for and preventing selected comorbidities in chronic inflammatory rheumatic diseases in daily practice: a EULAR initiative' by Baillet et al. Ann Rheum Dis 2016;75:e55

2 Baillet A, Gossec L, Carmona L, et al. Points to consider for reporting, screening for and preventing selected comorbidities in chronic inflammatory rheumatic diseases in daily practice: a EULAR initiative. Ann Rheum Dis 2016;75:965-73.

3 Wolfe F, Smythe HA, Yunus MB, et al. The American College of Rheumatology 1990 Criteria for the Classification of Fibromyalgia. Report of the Multicenter Criteria Committee. Arthritis Rheum 1990;33:160-72.

4 Wolfe F, Clauw DJ, Fitzcharles MA, et al. The American College of Rheumatology preliminary diagnostic criteria for fibromyalgia and measurement of symptom severity. Arthritis Care Res (Hoboken) 2010;62:600-10.

5 American College of Cardiology/American Heart Association Task Force on Practice Guidelines, Obesity Expert Panel, 2013. Guidelines for the management of overweight and obesity in adults: a report of the American College of Cardiology/ American Heart Association Task Force on Practice Guidelines and the Obesity Society published by the Obesity Society and American College of Cardiology/ American Heart Association Task Force on Practice Guidelines. Based on a systematic review from the The Obesity Expert Panel, 2013. Obesity 2014;22:55-39.

6 Bischoff-Ferrari HA, Willett WC, Wong JB, et al. Prevention of nonvertebral fractures with oral vitamin $D$ and dose dependency: a meta-analysis of randomized controlled trials. Arch Intern Med 2009;169:551-61.

7 Arends S, Spoorenberg A, Bruyn GA, et al. The relation between bone mineral density, bone turnover markers, and vitamin $\mathrm{D}$ status in ankylosing spondylitis patients with active disease: a cross-sectional analysis. Osteoporos Int 2011;22:1431-9.

8 Mitra D, Elvins DM, Collins AJ. Biochemical markers of bone metabolism in mild ankylosing spondylitis and their relationship with bone mineral density and vertebral fractures. J Rheumatol 1999;26:2201-4.

9 Gossec L, Baillet A, Dadoun S, et al. Collection and management of selected comorbidities and their risk factors in Chronic Inflammatory Rheumatic Diseases in daily practice in France. Ann Rheum Dis 2016. http://dx.doi.org10.1136/ annrheumdis-2016-eular.2885

10 Corrales A, Parra JA, González-Juanatey C, et al. Cardiovascular risk stratification in rheumatic diseases: carotid ultrasound is more sensitive than Coronary Artery Calcification Score to detect subclinical atherosclerosis in patients with rheumatoid arthritis. Ann Rheum Dis 2013;72:1764-70. 